\title{
Per 2000 Red Blood Cells
}

National Cancer Institute

\section{Source}

National Cancer Institute. Per 2000 Red Blood Cells. NCI Thesaurus. Code C132473.

Natural number unit of measurement for a portion of a particular type of cell (excluding red blood cell subtypes) per 2000 red blood cells. 